\title{
Multiband optical observations of the old PSR B0950+08 ^
}

\author{
S. V. Zharikov ${ }^{1}$, Yu. A. Shibanov ${ }^{2}$, R. E. Mennickent ${ }^{3}$, V. N. Komarova ${ }^{4,5}$, \\ A. B. Koptsevich ${ }^{2}$, and G. H. Tovmassian ${ }^{1}$
}

\author{
1 Observatorio Astronomico Nacional SPM, Instituto de Astronomia, UNAM, Ensenada, BC, Mexico \\ 2 Ioffe Physical Technical Institute, Politekhnicheskaya 26, St. Petersburg, 194021, Russia \\ e-mail: kopts@astro.ioffe.ru; shib@stella.ioffe.ru \\ 3 Departamento de Fisica, Universidad de Concepcion, Casilla 160, Concepcion, Chile \\ e-mail: rmennick@stars.cfm.udec.cl \\ ${ }^{4}$ Special Astrophysical Observatory Russian Academy of Science, Nizhnii Arkhyz 369167, Russia \\ e-mail: vkom@sao.ru \\ 5 Isaac Newton Institute of Chile, SAO Branch, Russia
}

Received 25 August 2003 / Accepted 5 December 2003

\begin{abstract}
We report on deep UBVRI-imaging of the field of the $17.3 \mathrm{Myr}$ radiopulsar PSR B0950+08 obtained with the ESO/VLT/FORS1. Firm detection of the candidate pulsar optical counterpart in the BVRI bands with the magnitudes $B=$ $27.06 \pm 0.35, V=27.05 \pm 0.15, R_{\mathrm{c}}=26.49 \pm 0.10$ and $I_{\mathrm{c}}=26.20 \pm 0.17$ enabled us to study, for the first time, the broadband spectrum of the object. The derived $\sim 0^{\prime} 24$ offset of the object from the radiopulsar position is insignificant within the 0.21 uncertainties of our astrometry and $\sim 0$ '.75 seeing value during the observations. The positional coincidence and unusual colors of the candidate ensure us that we likely detect the optical emission from PSR B0950+08. The optical-near-UV spectrum of the pulsar has a negative slope and can be fitted by a power law $F_{v} \propto v^{-\alpha}$ with $\alpha=0.65 \pm 0.40$, suggesting nonthermal emission. Within errors the optical flux is consistent with the power law fit of the ROSAT spectrum from the X-ray counterpart. We analyze our results together with the available multiwavelength data on other isolated pulsars of different ages detected in the optical range and find a significant correlation between their optical and 2-10 keV X-ray luminosities. This implies an origin of the nonthermal emission in both spectral domains. These objects show a significantly non-monotonic evolution of the efficiency of the optical-X-ray photon production from the pulsar spindown power, with a pronounced minimum at the beginning of the middle-age epoch and comparably high efficiencies of younger and older pulsars. This suggests different sensitivities of the photon production processes to the Goldreich-Julian current in magnetospheres of different age pulsars.
\end{abstract}

Key words. stars: pulsars: general - stars: pulsars: individual: PSR B0950+08 - stars: neutron

\section{Introduction}

Multiwavelength observations of radio pulsars are an important tool for the study of the not yet clearly understood radiative mechanisms and spectral evolution of rotation powered isolated neutron stars (NSs). With a canonical age of $\sim 1.7 \times 10^{7} \mathrm{yr}$, pulsar B0950+08 is of particular interest in this respect since it is the oldest among other ordinary pulsars detected outside the radio range (see for details Zharikov et al. 2002).

While the existence of the X-ray counterpart of PSR B0950+08 has been confirmed by different authors (Seward \& Wang 1988; Cordova et al. 1989; Manning \& Willmore 1994), its optical identification, suggested by Pavlov et al. (1996), remained doubtful for a long time. This was due to a poor coincidence of a faint, 27 . 1 , point-like source,

Send offprint requests to: $\mathrm{S}$. V. Zharikov,

e-mail: zhar@astrosen.unam.mx

* Based on observations collected at the European Southern Observatory, Paranal, Chile (ESO Programme 66.D-0363A). detected in the F130LP band with the HST/FOC in the near-UV range, with the radio pulsar position. The reported offset, $\sim 1^{\prime \prime} 85$, was significantly higher than the uncertainties (up to $1^{\prime \prime}$ ) of the Guide Star Catalog, which was only used for astrometric referencing of the 7". $4 \times 7$ ". $4 \mathrm{HST} / \mathrm{FOC}$ frame containing no other stars. Although the offset was later revised and decreased to $\sim 1^{\prime \prime}$ (Pavlov, private communication), it was still too large to ensure the positional coincidence of the optical object with the pulsar.

Further observations with the 6 m BTA telescope (Sokolov et al. 1998; Kurt et al. 2000) also revealed an optical object with $R=25.5 \pm 0.3$ in the pulsar field. Its offsets from the pulsar radio position and from the HST candidate were $\sim 1^{\prime \prime} .5 \pm 1^{\prime \prime}$. Within the astrometrical uncertainties one could not exclude that the HST and BTA detected the same source that this is the pulsar optical counterpart. However, in this case the optical spectrum of the PSR B0950+08 would have a very steep increase toward longer wavelengths. As other observed pulsar optical spectra are typically close to a flat spectrum, this 
Table 1. Parameters of PSR B0950+08 (Taylor et al. 1993, unless specified otherwise).

\begin{tabular}{|c|c|c|c|c|c|c|c|c|}
\hline \multicolumn{5}{|c|}{ Observed } & \multicolumn{4}{|c|}{ Derived } \\
\hline$P$ & $\dot{P}$ & $D M$ & $\alpha_{2000}, \delta_{2000}^{a}$ & $\mu_{\alpha}, \mu_{\delta}^{b}$ & $\tau$ & $B$ & $\dot{E}$ & $d^{b}$ \\
\hline $\mathrm{ms}$ & $10^{-15}$ & $\mathrm{~cm}^{-3} \mathrm{pc}$ & & mas $\mathrm{yr}^{-1}$ & Myr & $10^{12} \mathrm{G}$ & $\operatorname{ergs~s}^{-1}$ & $\mathrm{pc}$ \\
\hline 253 & 1.16 & 2.97 & $09^{\mathrm{h}} 53^{\mathrm{m}} 09^{\mathrm{s}} .314(3)^{c}$ & $-1.6(4)^{c}$ & 17.3 & 0.25 & $5.6 \times 10^{32}$ & $262(5)^{c}$ \\
\hline & & & $+07^{o} 55^{\prime} 36^{\prime \prime} 07(4)^{c}$ & $29.5(5)^{c}$ & & & & \\
\hline
\end{tabular}

\footnotetext{
${ }^{a}$ The radio position of the PSR B0950+08 at the epoch of the observations 2001.054 (MJD = 51 930).

${ }^{b}$ The pulsar proper motion and distance are from Brisken et al. (2002).

${ }^{c}$ Hereafter the numbers in parentheses are uncertainties referring to the last significant digits quoted, for example, $09^{\mathrm{h}} 53^{\mathrm{m}} 09^{\mathrm{s}} .314(3) \equiv 09^{\mathrm{h}} 53^{\mathrm{m}} 09^{\mathrm{s}} .314 \pm 0.003$ and $29.5(5) \equiv 29.5 \pm 0.5$.
}

deviation, if real, would be an extremely unusual departure from expected spectral behavior. This fact together with the short exposure time and low signal to noise ratio $(S / N=4.2)$ in addition to the poor seeing conditions of the BTA observations still kept the optical identification of the pulsar doubtful.

Recent subarcsecond imaging of the pulsar field obtained with the Subaru telescope in the $B$ band (Zharikov et al. 2002) and new more accurate radio measurements of the pulsar proper motion (Brisken et al. 2002) have considerably improved the situation. The Subaru observations led to the detection of a faint, $B=27.04 \pm 0.15$, optical object with coordinates coinciding with the radio coordinates of the pulsar with an accuracy better than $\sim 0{ }^{\prime} 4$. The Subaru image allowed revision of the HST/FOC astrometry using the FOC position angle and the only reference point $\sim 7^{\prime \prime}$ away of the pulsar, presumably a distant background galaxy, visible in both the Subaru and HST/FOC frames. As a result it was shown that the Subaru and HST candidates of the optical counterpart coincide within an accuracy of $\sim 0$ !'26 and are the same object. It is important that the positional coincidence is apparently better with the allowance for the pulsar proper motion during the $5.7 \mathrm{yr}$ between the HST and Subaru observations. This fact and the absence of any detectable source within at least $5^{\prime \prime}$ radius around the pulsar position, as well as a flatness of the spectrum of the object inferred from the HST and Subaru flux measurements strongly suggest that the detected object is likely to be the optical counterpart of PSR B0950+08.

This implies that the optical spectrum of the pulsar is of nonthermal origin. As follows from the measured optical fluxes and accurate radio measurements of the parallax-based distance to PSR B0950+08 (Brisken et al. 2002), the estimated efficiency of the optical photon production from the spin-down power of the pulsar is by several orders of magnitude higher than for middle-aged pulsars such as Geminga and PSR B0656+14 and comparable with that for the much younger and more energetic Crab pulsar. This is in contrast to a phenomenological conception of a monotonic decrease of the efficiency with pulsar age suggested earlier (Goldoni et al. 1995). The Subaru observations cannot exclude the presence of a compact, $\sim 1^{\prime \prime}$, faint pulsar nebula around PSR B0950+08, elongated perpendicular to the vector of its proper motion (for details see Zharikov et al. 2002 and Sect. 3.2). These results are crucial for the understanding of the evolution of the multiwavelength emission of pulsars, but they need to be confirmed by observations in other optical bands.

In this paper we continue to study PSR B0950+08 and report results of our broadband $U B V R I$ optical observations of the pulsar field obtained with the FORS1 at the VLT. The pulsar parameters (period $P$ and its derivative $\dot{P}$, age $\tau$, magnetic field $B$, dispersion measure $D M$, spindown luminosity $\dot{E}$, distance $d$, position $\alpha, \delta$, and proper motion $\mu_{\alpha}, \mu_{\delta}$ ) are listed in Table 1. We analyze our data together with the available optical-UV data from the HST, BTA, and Subaru, and with X-ray data from the ROSAT. Observations and data reduction are described in Sect. 2, in Sect. 3 we present the astrometrical referencing and photometry, and in Sect. 4 we discuss the results and their implications and summarize them in Sect. 5.

\section{Observations}

The field of PSR B0950+08 was observed during five nights from December 31, 2000-January 21, 2001 with the FORS1 (direct imaging mode) at the primary focus of the VLT-UT1 telescope using the service mode of observations. The FORS $1^{1}$ has a $2080 \times 2048 \mathrm{CCD}$ detector that provides $3.4^{\prime} \times 3.4^{\prime} \mathrm{FOV}$ or $\sim 0.1^{\prime \prime} /$ pixel spatial resolution with the high resolution collimator. The observations were carried out with the $U, B, V, R_{\mathrm{c}}$, $I_{\mathrm{c}}$ filters of the Johnson-Cousins system (Bessel 1990). The log of observations of the pulsar field and photometric standards are presented in Tables 2 and 3.

Standard data reduction including bias subtraction, flatfielding, and removing cosmic ray traces was performed making use of the ccdproc task of the NOAO IRAF software. The overview of the pulsar field as it is seen with the VLT in the $V$ band is shown in Fig. $1^{2}$.

\section{Astrometry and photometry}

\subsection{Astrometry}

The radio position of PSR B0950+08 at the epoch of the VLT observations (for which 19.01.2001 was adopted, see Table 1) was determined using the VLBA observations

\footnotetext{
see http: //www . eso.org/instruments/fors/

2 The images are available in the FITS format at http: //www . ioffe.ru/astro/NSG/obs/0950-vlt/
} 
Table 2. Log of the VLT/FORS1 observations of the PSR B0950+08 field in the UBVRI bands.

\begin{tabular}{|c|c|c|c|c|c|c|c|c|c|c|c|}
\hline $\begin{array}{l}\text { Date } \\
\text { UT }\end{array}$ & $\overline{\mathrm{UT}}$ & $\overline{\text { Band }}$ & $\begin{array}{c}\text { Exp. time } \\
\text { s }\end{array}$ & Airmass & $\begin{array}{l}\text { Seeing } \\
\operatorname{arcsec}\end{array}$ & $\begin{array}{l}\text { Date } \\
\text { UT }\end{array}$ & $\overline{\mathrm{UT}}$ & Band & $\begin{array}{c}\text { Exp. time } \\
\mathrm{S}\end{array}$ & Airmass & $\begin{array}{l}\text { Seeing } \\
\text { arcsec }\end{array}$ \\
\hline 31 Dec. 2000 & $07: 05$ & $R$ & 600 & 1.21 & 0.85 & 20 Jan. 2001 & $06: 21$ & $U$ & 600 & 1.18 & 0.64 \\
\hline -"- & $07: 16$ & $R$ & 600 & 1.20 & 0.80 & -"- & $06: 32$ & $U$ & 600 & 1.18 & 0.65 \\
\hline -"- & $07: 26$ & $R$ & 600 & 1.19 & 0.62 & -”- & $06: 43$ & $U$ & 600 & 1.18 & 0.81 \\
\hline -"- & $07: 37$ & $R$ & 600 & 1.18 & 0.64 & -”- & $06: 54$ & $U$ & 600 & 1.19 & 0.77 \\
\hline -”- & $07: 48$ & $R$ & 600 & 1.18 & 0.78 & -"- & 07:05 & $U$ & 600 & 1.19 & 0.71 \\
\hline -”- & $07: 59$ & $R$ & 750 & 1.18 & 0.78 & -”- & $07: 17$ & $B$ & 700 & 1.21 & 0.83 \\
\hline _”- & $08: 13$ & $R$ & 750 & 1.19 & 0.84 & -”- & 07:29 & $B$ & 700 & 1.22 & 0.70 \\
\hline -"- & $08: 26$ & $R$ & 750 & 1.20 & 0.81 & -”- & $07: 42$ & $B$ & 700 & 1.24 & 0.66 \\
\hline 1 Jan. 2001 & $06: 48$ & $R$ & 750 & 1.22 & 0.87 & -"- & $07: 55$ & $B$ & 700 & 1.27 & 0.63 \\
\hline -"- & 07:09 & $R$ & 750 & 1.20 & 0.92 & -”- & 08:07 & $B$ & 700 & 1.30 & 0.61 \\
\hline -”- & $07: 23$ & $R$ & 750 & 1.19 & 0.80 & -"- & $08: 20$ & $R$ & 600 & 1.34 & 0.60 \\
\hline _"- & $07: 36$ & $R$ & 750 & 1.18 & 0.92 & -"- & 08:31 & $R$ & 600 & 1.38 & 0.56 \\
\hline -"- & $07: 50$ & $R$ & 750 & 1.18 & 0.89 & -"- & $08: 42$ & $R$ & 600 & 1.42 & 0.65 \\
\hline -"- & 08:04 & $R$ & 600 & 1.19 & 0.88 & 21 Jan. 2001 & $04: 31$ & $I$ & 600 & 1.37 & 0.61 \\
\hline -”- & $08: 15$ & $R$ & 600 & 1.19 & 0.84 & -"- & $04: 42$ & $I$ & 600 & 1.33 & 0.61 \\
\hline -"- & $08: 26$ & $R$ & 600 & 1.20 & 0.74 & -"- & $04: 53$ & $I$ & 600 & 1.30 & 0.66 \\
\hline -"- & $08: 37$ & $R$ & 600 & 1.21 & 0.66 & -"- & 05:04 & $I$ & 600 & 1.27 & 0.65 \\
\hline 19 Jan. 2001 & $06: 22$ & V & 600 & 1.18 & 0.90 & -"- & $05: 15$ & $I$ & 600 & 1.25 & 0.79 \\
\hline -"- & $06: 33$ & V & 600 & 1.18 & 0.81 & -"- & $05: 26$ & V & 600 & 1.23 & 0.82 \\
\hline -"- & $06: 44$ & V & 600 & 1.18 & 0.82 & -"- & $05: 37$ & V & 600 & 1.21 & 0.77 \\
\hline -"- & $06: 55$ & V & 600 & 1.19 & 1.01 & -"- & $05: 55$ & V & 600 & 1.19 & 0.69 \\
\hline -"- & 07:06 & V & 600 & 1.19 & 1.12 & -"- & 06:06 & V & 600 & 1.19 & 0.65 \\
\hline -"- & $07: 22$ & $I$ & 600 & 1.21 & 0.82 & -"- & $06: 17$ & V & 600 & 1.18 & 0.71 \\
\hline -”- & $07: 33$ & $I$ & 600 & 1.22 & 0.75 & -"- & $06: 29$ & $I$ & 600 & 1.18 & 0.63 \\
\hline -"- & $07: 44$ & $I$ & 600 & 1.24 & 0.81 & -"- & $06: 40$ & $I$ & 600 & 1.18 & 0.54 \\
\hline -"- & $07: 55$ & $I$ & 600 & 1.26 & 0.76 & -"- & $06: 51$ & $I$ & 600 & 1.19 & 0.62 \\
\hline \multirow[t]{2}{*}{-"- } & 08:06 & $I$ & 600 & 1.29 & 0.70 & -"- & 07:02 & $I$ & 600 & 1.19 & 0.60 \\
\hline & & & & & & -"- & $07: 13$ & $I$ & 600 & 1.21 & 0.54 \\
\hline
\end{tabular}

Table 3. Log of the VLT/FORS1 observations of the photometrical standards used for the calibration.

\begin{tabular}{ccccccc}
\hline \hline $\begin{array}{c}\text { Date } \\
\text { UT }\end{array}$ & Name & UT & Band & $\begin{array}{c}\text { Exp. time } \\
\text { s }\end{array}$ & Airmass & $\begin{array}{c}\text { Seeing } \\
\text { arcsec }\end{array}$ \\
\hline 01 Jan. 2001 & PG1047+003 & $08: 55$ & $R$ & 1 & 1.10 & 0.65 \\
20 Jan. 2001 & PG0942-029 & $09: 01$ & $U$ & 80 & 1.37 & 0.65 \\
20 Jan. 2001 & PG0942-029 & $09: 04$ & $B$ & 6 & 1.39 & 0.65 \\
20 Jan. 2001 & PG0942-029 & $09: 05$ & $V$ & 3 & 1.39 & 0.65 \\
20 Jan. 2001 & PG0942-029 & $09: 06$ & $R$ & 3 & 1.40 & 0.65 \\
20 Jan. 2001 & PG0942-029 & $09: 07$ & $I$ & 6 & 1.40 & 0.65 \\
21 Jan. 2001 & RU149 & $04: 20$ & $U$ & 60 & 1.09 & 0.65 \\
21 Jan. 2001 & RU149 & $04: 22$ & $B$ & 2 & 1.10 & 0.65 \\
21 Jan. 2001 & RU149 & $04: 23$ & $V$ & 2 & 1.10 & 0.65 \\
21 Jan. 2001 & RU149 & $04: 24$ & $R$ & 2 & 1.10 & 0.65 \\
21 Jan. 2001 & RU149 & $04: 25$ & $I$ & 2 & 1.10 & 0.65 \\
21 Jan. 2001 & RU149 & $07: 29$ & $U$ & 60 & 1.79 & 0.65 \\
21 Jan. 2001 & RU149 & $07: 32$ & $B$ & 2 & 1.81 & 0.65 \\
21 Jan. 2001 & RU149 & $07: 33$ & $V$ & 2 & 1.83 & 0.65 \\
21 Jan. 2001 & RU149 & $07: 34$ & $R$ & 2 & 1.84 & 0.65 \\
21 Jan. 2001 & RU149 & $07: 35$ & $I$ & 2 & 1.85 & 0.65 \\
\hline
\end{tabular}

(Fomalont et al. 1992) and recent measurements of the pulsar proper motion (Brisken et al. 2002).

Astrometrical referencing of the VLT images was made using positions of reference stars from the USNO B1.0 catalog (0979-0228656, 0979-0228669, 0979-0228673, 0979-0228679, 0979-0228696, 0979-0228699,0979-0228707) visible within the FORS1 frames. These stars are marked by crosses in Fig. 1. 


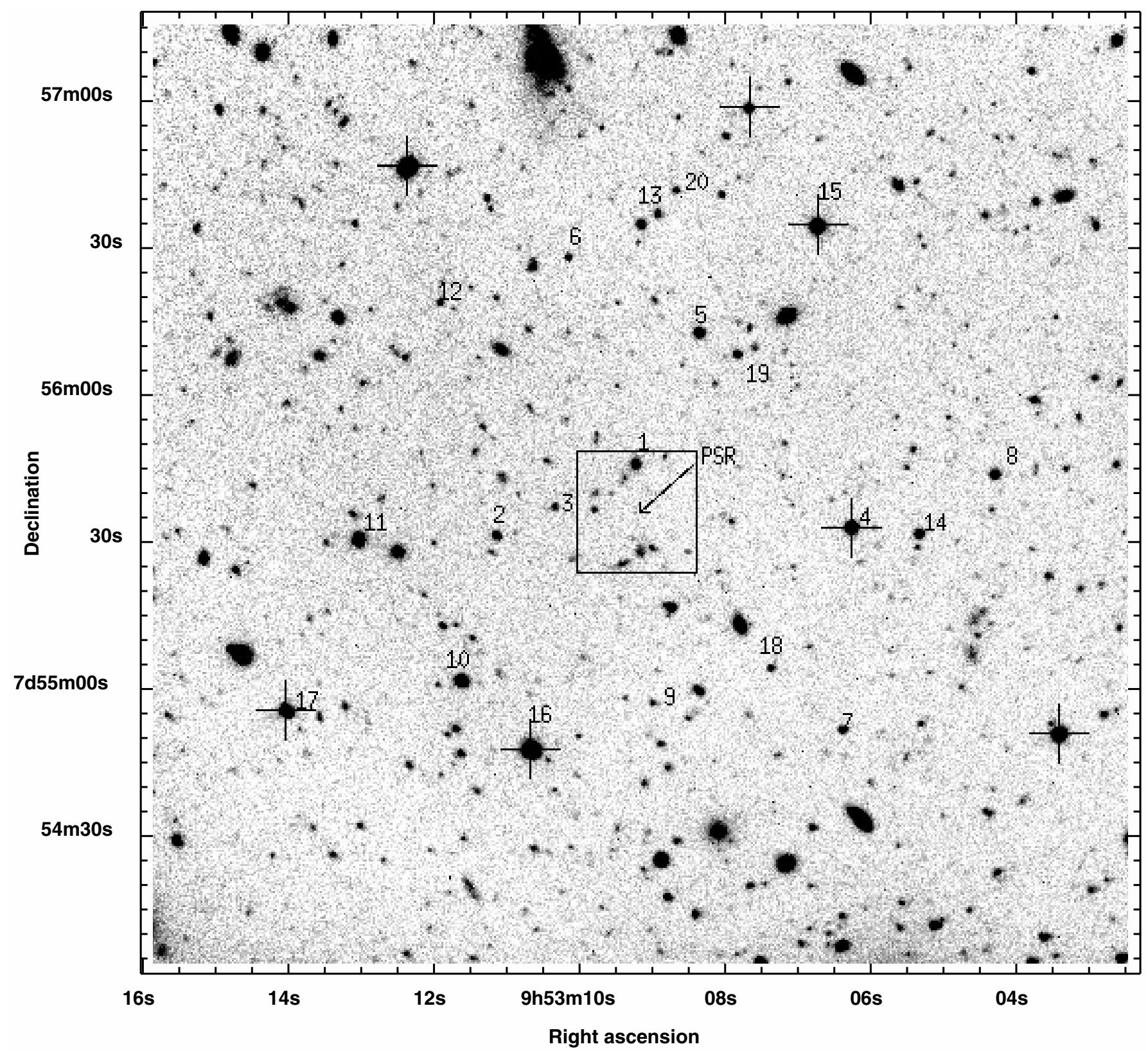

Fig. 1. The VLT image of the PSR B0950+08 field in the $V$ band with an accumulated exposure time of $6000 \mathrm{~s}$. The center of $25^{\prime \prime} \times 25^{\prime \prime}$ box coincides with the pulsar position shown by the arrow. The fragment within the box is blown up in Fig. 2. North is up, East is left. Numbers denote the objects whose coordinates and magnitudes are given in Table 4. Crosses mark the USNO B1.0 catalog stars used for astrometric referencing of the VLT images.

All images were aligned using a set of bright unsaturated stars and the IRAF tasks ccmap/cctran were applied for the astrometrical transformation of the image obtained from the sum of all color data. The formal rms errors of the astrometric fit were $\Delta \alpha=0 .{ }^{\prime} 011$, and $\Delta \delta=0{ }^{\prime} 046$, while the residuals for all reference stars are $\leq 0{ }^{\prime} 08$. These are less than the nominal uncertainties of the USNO B1.0 catalog $\left(0{ }^{\prime} 2\right)$ and the mean seeing value during our observations (0.75). By combining in a standard way the rms errors of the fit, the USNO cata$\log$ uncertainties and the radio position errors (see Table 1) we derived a secure $1 \sigma$ uncertainty of the pulsar position in our images, as well as the astrometric referencing accuracy of the whole VLT images, as 0!'21 in RA and Dec. This is within the uncertainty of the astrometric referencing of the much wider FOV optical image of the PSR B0950+08 field obtained by Zharikov et al. (2002) with the Subaru/Suprime-Cam using a different set of the USNO stars.

\subsection{Identification of the pulsar counterpart}

In Fig. 2 we present the $25^{\prime \prime} \times 25^{\prime \prime}$ fragments of the BVRI images (left panels) constrained by the box in Fig. 1. We show also the contour maps of the $7^{\prime \prime} \times 7^{\prime \prime}$ regions centered within the fragments (middle panels), and the magnification of the 1 .'5 $\times 1$ ". 5 part of the counter map in the $B$ band containing the pulsar (right-up panel). The images and maps are smoothed 

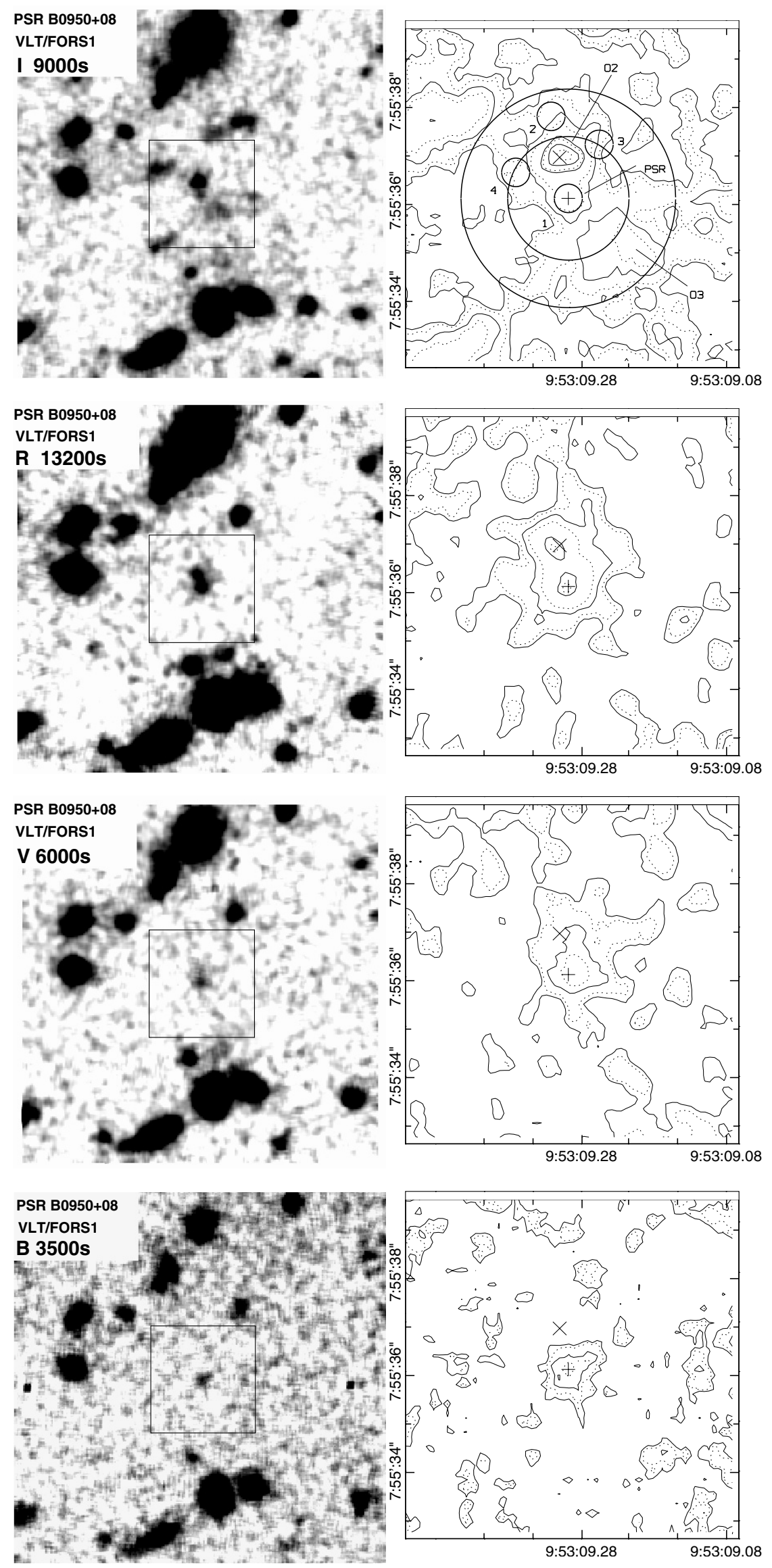

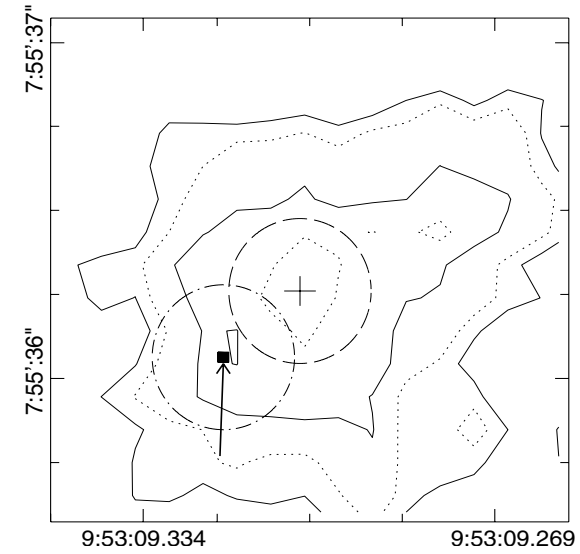

Fig. 2. Left panels: $25^{\prime \prime} \times 25^{\prime \prime}$ fragments of the BVRI images of the PSR B0950+08 field. The images are smoothed over the area of $2 \times 2$ pixels. Middle panels: the contour maps of the 7" $\times 7$ " box shown in the images in the left panels. The candidate pulsar optical counterpart (PSR) and two nearby red extended objects $\mathrm{O} 2$ and $\mathrm{O} 3$ are indicated in the $I$ band map. "+" and " $x$ " mark the Gaussian fitted positions of the candidate and O2, respectively. Small and large rings show the apertures and background annuli used for the candidate photometry (see Sect. 3.4). Right-upper panel: magnification of the $1^{\prime \prime} .5 \times 1$ ". 5 region of the $B$ band contour map containing the pulsar. The filled square and dot-dashed ellipse show the radio pulsar position and its $1 \sigma$ uncertainty. The dashed ellipse marks $1 \sigma$ uncertainty of the candidate position. The arrow indicates the direction of the pulsar proper motion, and its length corresponds to the path of the pulsar over the last $10 \mathrm{yr}$. 
over the areas of $2 \times 2$ pixels making use of the ESO-MIDAS filter/gauss procedure. The isophotes in the maps correspond to the flux levels $l_{n}$ (in counts) above the background:

$l_{n}=S+n k \sigma$,

where $S$ is the mean sky value near the pulsar; $n=0,1,2, \ldots, N$; $\sigma$ is the sky standard deviation related to one pixel; $k$ is the scaling factor. We used $k=0.1$. The radio position of the pulsar is marked by a filled square in the $B$ band map.

The candidate PSR B0950+08 optical counterpart suggested by Pavlov et al. (1996) and later confirmed by Zharikov et al. (2002) based on the HST/FOC and Subaru/Suprime-Cam observations in the near-UV F130LP and $B$ bands is clearly visible in the centers of the VLT $B V R I$ images. In addition, we detected two faint extended red objects, $\mathrm{O} 2$ and $\mathrm{O} 3$, only $\leq 1^{\prime \prime}$ north and south-south-west of the pulsar, respectively. Both objects are not visible in the $B$ band and $\mathrm{O} 3$ is fainter and seen only in the $I$ band. No objects were detected within a $7^{\prime \prime} \times 7^{\prime \prime}$ box centered at the pulsar position during a $3000 \mathrm{~s}$ exposure in the $U$ band and we do not show it here.

Seeing conditions and a low $S / N \simeq 3-4.5$, related to a peak pixel value depending on the band, do not allow us to resolve any point-like source in the VLT candidate profile, while the object has been definitely detected as a point-like source with the HST/FOC. Nevertheless, a formal Gaussian fitting of the VLT source profile in the $B$ band, where the object is not contaminated by the nearby $\mathrm{O} 2$, yields the position $\alpha_{2000}=09^{\mathrm{h}} 53^{\mathrm{m}} 09^{\mathrm{s}} .305(17), \delta_{2000}=+7^{\circ} 55^{\prime} 36^{\prime \prime} \cdot 27(24)$. This position is marked by " + " in the counter maps. Its $1 \sigma$ uncertainty ellipse overlaps the respective ellipse of the pulsar radio position, shown in the enlarged counter map in the right-upper panel of Fig. 2, and consistent with the position derived earlier from the Subaru observation (Zharikov et al. 2002). The $\mathrm{N}-\mathrm{W}$ offset from the radio position of the pulsar is $\cong 0.24$ ( $\Delta \alpha \cong 00^{\prime} 14, \Delta \delta \cong 0.2$ ) and can be considered as insignificant within the $\sim 0$. 75 seeing and $\sim 0$.'21 uncertainty of our astrometric referencing. It is about half the size of the $\mathrm{W}-\mathrm{N}$ offset derived from the Subaru image (Zharikov et al. 2002), which may be due to a systematic astrometric error caused by larger uncertainties of the USNO A1.0 catalog, available at that time, and the absence of any reference star south of the the pulsar in the Subaru image. Thus, our VLT observations confirm and improve the positional coincidence of the suggested optical counterpart with the pulsar. Consistent with the Subaru observations (Zharikov et al. 2002) the outer contour of the candidate source profile in the VLT B image is slightly ( 20\%) elongated in the direction approximately perpendicular to the direction of the pulsar proper motion shown by an arrow in the right-up panel of Fig. 2. Detection of the elongation with the two different telescopes suggests that it is real. Deeper observations with higher spatial resolution are necessary to study its morphology and spectral energy distribution to determine whether it is associated with a faint pulsar wind nebula (PWN) or with a background object. In this context we note that wings of spatial profiles of brighter optical counterparts of two middle-aged pulsars PSR B0656+14 and Geminga are also poorly consistent with a point spread function (Komarova et al. 2003), that may be due to the presence of a faint few arcseconds PWN around each pulsar.

The coordinates of $\mathrm{O} 2$ derived from the $I$ band image are $\alpha_{2000}=09^{\mathrm{h}} 53^{\mathrm{m}} 09^{\mathrm{s}} .312(26)$ and $\delta_{2000}=+7^{\circ} 55^{\prime} 37^{\prime \prime}$.04(40). Its position is marked by " $\times$ " in the contour maps. The coordinates of the diffuse source $\mathrm{O} 3$ barely seen in the $I$ band cannot be definitely determined from our data.

\subsection{Photometric referencing}

The photometric conditions were rather stable during the VLT observations. Photometric referencing was carried out with the PG1047+003, PG0942-029, RU149 photometric standards from Landolt (1992). From the variation of count rates of the RU149 field stars with the airmass observed on the night of Jan. 21, 2001 (see the Log in Table 3) we derived the atmospheric extinction factors in the $U B V R I$ bands $k_{U}=0.50 \pm 0.03$, $k_{B}=0.29 \pm 0.02, k_{V}=0.15 \pm 0.01, k_{R}=0.11 \pm 0.01$, $k_{I}=0.06 \pm 0.03$. This is in agreement with the mean values obtained for the VLT site ${ }^{3}$.

The extinction factors and the magnitude measurements of the RU 149 standard stars led to the following transition equations

$$
\begin{aligned}
U-u & =24.149(87)+0.114(32) \cdot(u-b) \\
B-b & =27.086(8)-0.081(24) \cdot(b-v) \\
V-v & =27.412(8)+0.030(8) \cdot(b-v) \\
V-v & =27.426(6)+0.068(19) \cdot(v-r) \\
R-r & =27.375(13)+0.043(44) \cdot(v-r) \\
R-r & =27.414(29)+0.052(48) \cdot(r-i) \\
I-i & =26.500(35)-0.077(57) \cdot(r-i)
\end{aligned}
$$

and

$$
\begin{aligned}
& U-B=-3.098(88)+1.199(33) \cdot(u-b) \\
& B-V=-0.331(14)+0.887(14) \cdot(b-v) \\
& V-R=0.470(1)+1.056(3) \cdot(v-r) \\
& R-I=0.899(18)+1.097(29) \cdot(r-i)
\end{aligned}
$$

where $(u, b, v, r, i)$ are the instrumental magnitudes and $U B V R I$ are the magnitudes in the Johnson-Cousins photometric system. The rms errors of the transformation coefficients shown in the parentheses include the magnitude errors of the standards and the instrumental magnitude errors, as well as the uncertainties of the extinction factors and of the calibration fit. Variations of the transformation coefficients from night to night based on our standards observational set (see Table 3 ) were estimated to be within $2 \sigma$ uncertainties of the coefficients in Eqs. (1) and (2).

The absolute fluxes $F_{j}$ (in ergs $\mathrm{cm}^{-2} \mathrm{~s}^{-1} \mathrm{~Hz}^{-1}$ ), where $j$ is the band name, were calculated using measured magnitudes $M_{j}$ and the equations

$\log F_{j}=-0.4\left(M_{j}+M_{j}^{0}\right)$,

with the zero-points provided by Fukugita et al. (1995): $M_{U}^{0}=$ 49.30, $M_{B}^{0}=48.49, M_{V}^{0}=48.613, M_{R}^{0}=48.80, M_{I}^{0}=$ 49.058 .

\footnotetext{
3 see http://www.eso.org/observing/dfo/quality/ index_fors 1 .html
} 


\subsection{Flux measurements of the PSR B0950+08 candidate}

The instrumental magnitudes of the optical counterpart of the pulsar in the $B$ band, where it is not contaminated by the nearby object $\mathrm{O} 2$, were measured for a range of aperture radii of (1-4) CCD pixels centered on the "+" in Fig. 2. The background was measured over the annulus with the inner and outer radii $r_{\text {in }}=13$ and $r_{\text {out }}=23$ pixels, as shown in the $I$ band contour map in Fig. 2. The magnitudes were corrected for the PSF of bright stars (details of our faint object photometry technique can be found in Kurt et al. 1998 and Koptsevich et al. 2001). Within the measurement accuracy the results for different apertures coincided and a 3 pixel radius was used as optimal based on the maximal $S / N$ ratio. The resulting flux in the $B$ band (see Table 5) actually coincides with that obtained by Zharikov et al. (2002) with the Subaru telescope at higher $S / N, F_{B}=0.0597 \pm$ $0.0088 \mu \mathrm{Jy}$. The contribution of the elongated source wings was estimated to be within the measured flux errors.

As seen from the contour maps in Fig. 2, the flux contamination of the PSR B0950+08 candidate by the wings of the extended $\mathrm{O} 2$ can be significant in $V$ and particularly in the $R I$ bands. The magnitudes of $\mathrm{O} 2$ are $B \lesssim 28, V \simeq 28, R \simeq 26.3$, and $I \simeq 24.8$.

This is a very red object. Its spectral energy distribution can be approximated by a power law $\left(\mathrm{F}_{v} \propto v^{-\alpha_{v}}\right)$ with a spectral index $\alpha_{v} \sim 6.45 \pm 0.55$. It could be considered as a PWN candidate. However, the spectral slope of $\mathrm{O} 2$ is much steeper than the spectral slopes of the Crab PWN structures $\left(\alpha_{v} \lesssim 0.8\right)$ detected in the optical and near-IR ranges (Sollerman 2003). In this respect a possible association of $\mathrm{O} 2$ with a PWN appears to be unplausible. Most likely $\mathrm{O} 2$ is a distant background galaxy, as observed in known Deep Field images. The situation is typical of deep optical studies of pulsar fields. For instance, the optical counterpart of PSR B0656+14 is also only $\sim 1$ " away from a faint red extended object (Kurt et al. 1998). A deep near-IR imaging has revealed the morphology the object and showed that it is likely to be a background spiral galaxy seen edge-on (Koptsevich et al. 2001). Current observations of the PSR B0950+08 field in other spectral domains are not yet deep enough to identify $\mathrm{O} 2$ and its nature.

If $\mathrm{O} 2$ had a point-like profile or its angular sizes were smaller than seeing, the contamination of the pulsar flux would be only about $3 \%$ and $4 \%$ in the $R$ and $I$ bands, respectively. To get more secure estimates of the contamination we used an approach similar to that suggested by Koptsevich et al. (2001) for the photometry of PSR B0656+14. We estimated the azimuth averaged flux from $\mathrm{O} 2$ near the pulsar position and subtracted this value from the flux measured in the aperture centered on the pulsar position. To do that we measured the fluxes from four apertures of 3 pixel radii (as we used above for the $B$ band). These apertures were placed symmetrically around the center of $\mathrm{O} 2$ at the radius of $0{ }^{\prime} 9$, which is equal to the distance between the center positions of $\mathrm{O} 2$ and the counterpart candidate. The aperture positions are shown in the $I$ band contour map of Fig. 2. The aperture number 1 is centered on the pulsar candidate position, number 2 is at the extension of the line connecting the candidate and $\mathrm{O} 2$, and $3 \mathrm{~d}$ and 4 th apertures are placed symmetrically at the perpendicular to this line. The optical counterpart flux was calculated as

$F_{\mathrm{PSR}}=F_{1}-\frac{F_{2}+F_{3}+F_{4}}{3}$

where the subscripts correspond to the aperture numbers. As a result, we obtained $(12 \pm 8) \%$ contamination of the candidate flux by $\mathrm{O} 2$ in $V,(10 \pm 7) \%$ in $R$, and $(27 \pm 11) \%$ in $I$ bands (see Table 5). The errors correspond to the mean deviations of the azimuth distribution of the $\mathrm{O} 2$ source profile from the respective value averaged over the azimuth at $0 \prime^{\prime} 9$ distance of the source center. The wings of the source are apparently brighter in the $\mathrm{N}-\mathrm{W}-\mathrm{W}$ direction (aperture number 3 in Fig. 2). Increasing the number of the symmetrically placed apertures does not change considerably our estimates. Checking the approach by applying it to the $B$ band image shows no contamination in this band, as expected.

The results of our photometry of the counterpart are presented in Table 5. They can be only improved by observations with higher spatial resolution provided by the HST and/or the VLT Interferometer, or by other large ground based telescopes equipped with Adaptive Optics systems. We estimated also the $3 \sigma$ upper limit of the object flux in the $U$ band as $U \lesssim 26.5$. This is consistent with the known flux in the HST/F130LP filter (Pavlov et al. 1996), whose long band pass overlaps the $U$ band.

Using a flat extrapolation of a mean flux over the $B V$ bands $(\sim 0.0525 \mu \mathrm{Jy})$ toward the $U$ band and the VLT exposure time calculator ${ }^{4}$ we obtain $\gtrsim 1.5 \mathrm{~h}$ exposure for a sure detection of the object in the $U$ band with telescopes like the VLT.

The object $\mathrm{O} 2$ is by a magnitude brighter in the $R I$ bands than the pulsar counterpart. This object and the pulsar candidate were not resolved in the BTA $R$ band images obtained in worse seeing conditions (Sokolov et al. 1998; Kurt et al. 2000). Hence both objects contributed to the measured flux suggested to be from the counterpart candidate. The reported BTA magnitude, $R \sim 25.5$, is in agreement with the value measured by us with the VLT.

As a by-product the field photometry was done. The magnitudes of twenty relatively bright stars indicated by numbers in Fig. 1, which can be used as secondary photometric standards in further optical observation of the pulsar field, are given in Table 4. In comparison with these stars our pulsar candidate shows significantly unusual colors, as other more luminous pulsars detected in the optical range. At the same time, a simple color-color analysis has not allowed us to distinguish our faint candidate from a hundred faint background objects of a similar brightness (with magnitudes of 26.5-28) seen in our VLT frames. Most of them are likely to be extragalactic objects, as in known Deep Field images. Much work has to be done for their classification and selection of galactic and extragalactic objects to understand whether it is possible to use color-color analysis of faint objects in deep images for identification of optical counterparts of isolated NSs. This is outside the scope of this paper.

\footnotetext{
${ }^{4}$ http://www.eso.org/observing/etc/index.html
} 
Table 4. The coordinates and stellar magnitudes of 20 stars around the pulsar marked in Fig. 1.

\begin{tabular}{cccccccc}
\hline \hline NN & $\alpha_{2000}$ & $\delta_{2000}$ & $\begin{array}{c}U \\
\left(\lambda_{\text {eff }}=3652 \AA,\right. \\
F W H M=526 \AA)\end{array}$ & $\begin{array}{c}B \\
\left(\lambda_{\text {eff }}=4448 \AA,\right. \\
F W H M=1008 \AA)\end{array}$ & $\begin{array}{c}V \\
\left(\lambda_{\text {eff }}=5505 \AA,\right. \\
F W H M=827 \AA)\end{array}$ & $\begin{array}{c}R \\
\left(\lambda_{\text {eff }}=6588 \AA,\right. \\
F W H M=1568 \AA)\end{array}$ & $\begin{array}{c}I \\
\left(\lambda_{\text {eff }}=8060 \AA\right. \\
F W H M=1542 \AA)\end{array}$ \\
\hline 1 & $09: 53: 09.3$ & $07: 55: 46.2$ & $24.96(21)$ & $24.03(3)$ & $22.52(1)$ & $21.38(5)$ & $21.56(6)$ \\
2 & $09: 53: 11.2$ & $07: 55: 31.7$ & $24.65(17)$ & $24.10(3)$ & $22.45(1)$ & $21.24(5)$ & $21.44(6)$ \\
3 & $09: 53: 10.4$ & $07: 55: 37.4$ & $23.44(10)$ & $24.22(3)$ & $23.73(1)$ & $23.04(5)$ & $22.82(6)$ \\
4 & $09: 53: 06.3$ & $07: 55: 33.1$ & $20.36(9)$ & $20.73(3)$ & $20.37(1)$ & $19.98(5)$ & $19.48(6)$ \\
5 & $09: 53: 08.4$ & $07: 56: 12.5$ & $23.76(11)$ & $22.91(3)$ & $21.38(1)$ & $20.10(5)$ & $20.37(6)$ \\
6 & $09: 53: 10.2$ & $07: 56: 28.0$ & $25.56(36)$ & $24.90(4)$ & $23.24(1)$ & $21.68(5)$ & $22.20(6)$ \\
7 & $09: 53: 06.5$ & $07: 54: 52.2$ & $22.82(10)$ & $23.04(3)$ & $22.57(1)$ & $22.09(5)$ & $21.67(6)$ \\
8 & $09: 53: 04.3$ & $07: 55: 43.9$ & $22.22(9)$ & $22.60(3)$ & $21.61(1)$ & $20.95(5)$ & $20.68(6)$ \\
9 & $09: 53: 09.1$ & $07: 54: 57.7$ & $23.95(12)$ & $24.53(3)$ & $24.10(1)$ & $23.45(5)$ & $23.15(6)$ \\
10 & $09: 53: 11.7$ & $07: 55: 02.1$ & $23.09(10)$ & $22.06(3)$ & $20.44(1)$ & $19.09(5)$ & $19.42(6)$ \\
11 & $09: 53: 13.1$ & $07: 55: 30.9$ & $22.01(9)$ & $22.35(3)$ & $21.46(1)$ & $20.79(5)$ & $20.54(6)$ \\
12 & $09: 53: 12.0$ & $09: 56: 18.9$ & $22.87(10)$ & $23.81(3)$ & $23.61(1)$ & $23.17(5)$ & $22.68(6)$ \\
13 & $09: 53: 09.2$ & $07: 56: 34.7$ & $24.35(15)$ & $23.41(3)$ & $21.78(1)$ & $20.30(5)$ & $20.76(6)$ \\
14 & $09: 53: 05.4$ & $07: 55: 37.8$ & $24.31(15)$ & $23.47(3)$ & $21.88(1)$ & $20.68(5)$ & $20.87(6)$ \\
15 & $09: 53: 06.7$ & $07: 56: 34.3$ & $22.04(9)$ & $21.08(3)$ & $19.57(1)$ & $18.54(5)$ & $18.58(6)$ \\
16 & $09: 53: 10.8$ & $07: 54: 48.3$ & $18.75(9)$ & $19.16(3)$ & $18.82(1)$ & $18.38(5)$ & $17.93(6)$ \\
17 & $09: 53: 14.1$ & $07: 54.56 .2$ & $20.14(9)$ & $20.49(3)$ & $20.19(1)$ & $19.76(5)$ & $19.31(6)$ \\
18 & $09: 53: 07.5$ & $07: 55: 04.7$ & $23.27(10)$ & $23.96(3)$ & $23.42(1)$ & $22.69(5)$ & $22.48(6)$ \\
19 & $09: 53: 07.9$ & $07: 56: 08.3$ & $22.93(10)$ & $23.13(3)$ & $22.48(1)$ & $21.97(5)$ & $21.57(6)$ \\
20 & $09: 53: 08.1$ & $07: 56: 40.6$ & $24.11(13)$ & $24.46(3)$ & $23.71(1)$ & $23.11(5)$ & $22.79(6)$ \\
\hline
\end{tabular}

Table 5. Flux measurements of the pulsar optical counterpart (see Sect. 3.4 for details).

\begin{tabular}{c|ccccc|cc|cc}
\hline \hline Band & $\begin{array}{c}F_{1}^{a} \\
\mathrm{DN} \mathrm{s}^{-1}\end{array}$ & $\begin{array}{c}F_{2}^{a} \\
\mathrm{DN} \mathrm{s}^{-1}\end{array}$ & $\begin{array}{c}F_{3}^{a} \\
\mathrm{DN} \mathrm{s}^{-1}\end{array}$ & $\begin{array}{c}F_{4}^{a} \\
\mathrm{DN} \mathrm{s}^{-1}\end{array}$ & $\begin{array}{c}F_{\text {PSR }} \\
\mathrm{DN} \mathrm{s}^{-1}\end{array}$ & $\begin{array}{c}k / \cos (Z)^{b} \\
\mathrm{mag}\end{array}$ & $\begin{array}{c}\delta_{\text {fin }} d^{c} \\
\mathrm{mag}\end{array}$ & Mag $^{d}$ & $\begin{array}{c}\text { Flux } \\
\mu \mathrm{Jy}^{2}\end{array}$ \\
\hline$B$ & 0.240 & 0.0 & 0.014 & 0.0 & 0.235 & 0.35 & 1.21 & $27.06(35)$ & $0.0602(166)$ \\
$V$ & 0.375 & 0.069 & 0.053 & 0.0 & 0.334 & 0.19 & 1.37 & $27.05(15)$ & $0.0448(57)$ \\
$R$ & 0.570 & 0.0 & 0.159 & 0.0 & 0.517 & 0.14 & 1.49 & $26.48(10)$ & $0.0909(80)$ \\
$I$ & 0.501 & 0.125 & 0.152 & 0.041 & 0.396 & 0.07 & 1.27 & $26.21(17)$ & $0.0784(113)$ \\
\hline
\end{tabular}

${ }^{a}$ Source count rates within the apertures $1-4$ shown in the $I$ band contour map in Fig. 2.

${ }^{b}$ Corrections for the atmospheric extinction.

${ }^{c}$ Correction factors for the PSF.

${ }^{d}$ Johnson-Cousins magnitudes.

\section{Discussion}

Positional coincidence of the counterpart candidate and the radiopulsar and its unusual colors suggest that we have detected the optical emission from PSR B0950+08. Assuming that, in Fig. 3 we combined our results with the available optical-UV data obtained with the HST and Subaru. We neglected the interstellar extinction, which is expected to be very low for this pulsar, $E_{B-V} \lesssim 0.02$ (Pavlov et al. 1996), and insignificant for our analysis.

\subsection{Nonthermal origin of the PSR B0950+08 optical emission}

As seen from Fig. 3, the broadband spectrum of the pulsar in the optical range does not follow the Rayleigh-Jeans law suggested by Pavlov et al. (1996) to explain the optical-UV radiation in the only HST/F130LP band as a low temperature thermal emission from the entire surface of a reheating/cooling NS (dashed line in Fig. 3). The latter contradicts also the X-ray observations, showing an order of magnitude lower X-ray flux in the ROSAT band than expected from the thermal emission with the temperature inferred from the Rayleigh-Jeans fit (Zharikov et al. 2002). Contrary to the thermal model, the optical spectrum demonstrates a negative slope. It is consistent with a single power law fit $F_{v} \propto v^{-\alpha_{v}}$ with $\alpha_{v}=0.65 \pm 0.40$ (solid line in Fig. 3). This suggests the nonthermal nature of the detected emission likely originating in the magnetosphere of the rotating NS. This fit is in agreement with the power law fit of the ROSAT X-ray data (Manning \& Willmore 1994).

The latter would imply a common origin of the nonthermal X-ray and the optical emission of PSR B0950+08, as it is likely to be for younger pulsars PSR B0656+14 (Koptsevich et al. 2001; Komarova et al. 2003) and Vela (Shibanov et al. 2003; Mignani \& Caraveo 2001). However, the ROSAT X-ray data on such a faint X-ray source as PSR B0950+08 are extremely limited and respective spectral information is much less certain (e.g., $\alpha_{v}=0.9(+1.2,-0.7)$ in the $0.08-2.4 \mathrm{keV}$ range (Manning \& Willmore 1994) than the optical one to make definite conclusions. 


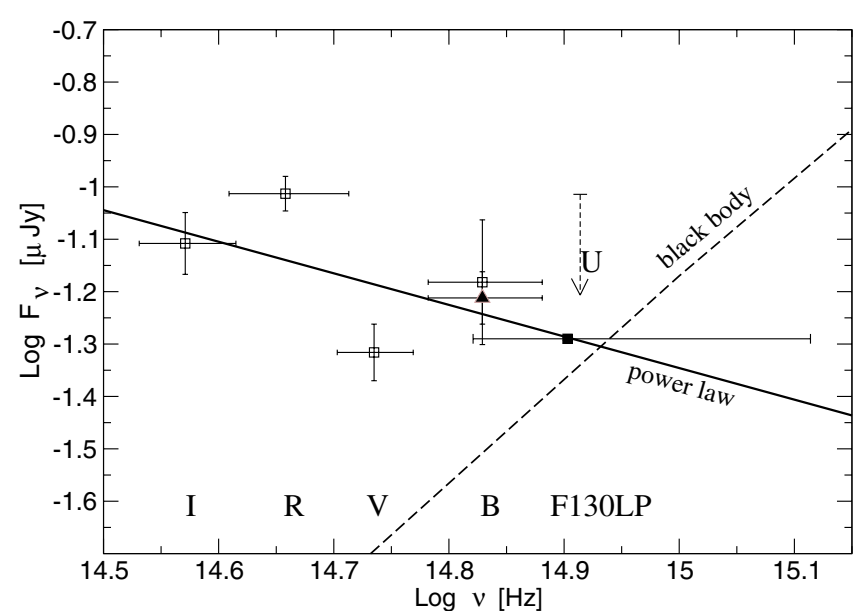

Fig. 3. Broadband optical spectrum of PSR B0950+08. The fluxes in the HST/F130LP (filled rectangular) and Subaru/B (filled triangle) bands are taken from Pavlov et al. (1996) and Zharikov et al. (2002), respectively. Solid line is the power law fit of the whole optical data with the spectral index $\alpha_{v}=0.65$. Dashed line (black body) is the Rayleigh-Jeans fit suggested by Pavlov et al. (1996) to explain the flux in the only F130LP band as thermal emission from the entire surface of the NS.

Some deviations from the single power law optical fit at about $2 \sigma$ level are seen in the $V$ and $R$ bands. They could be due to unresolved spectral features associated either with the ion cyclotron emission/absorption near the surface of the strongly magnetized NS, as was suggested for Geminga by Martin et al. (1998), or with an unresolved pulsar nebula, as was detected in a grism spectrum of PSR B0540-69 by Hill et al. (1997). But at this moment we not can exclude that the deviations are caused by an inaccurate account of the pulsar flux contamination by the nearby extended object $\mathrm{O} 2$. Better spatial resolution observations are necessary to ensure that the deviations are significant, as has been shown in case of PSR B0656+14 (Koptsevich et al. 2001; Komarova et al. 2003).

\subsection{Optical spectrum of PSR B0950+08 and spectral evolution of the pulsar optical emission}

The VLT observations expand significantly the broadband optical spectrum of PSR B0950+08 toward longer wavelengths. This allows a comparison with the spectral properties of other pulsars detected in the optical range. In Fig. 4 we compare our results with available dereddened phase-averaged optical spectra of pulsars of different ages. In the $B$ band for PSR B0950+08 we use the more accurate flux value obtained with the Subaru (Zharikov et al. 2002).

The reliable spectral information is still available only for the youngest and brightest Crab pulsar, whose spectrum has a positive slope and monotonously increases from the nearIR to UV range (Sollerman et al. 2000; Sollerman 2003). In contrast to that, the broadband spectrum of the ten times older Vela pulsar appears to have a negative slope (Shibanov et al. 2003). The broadband spectra of the middle-aged pulsars PSR B0656+14 and Geminga are less monotonic and have dips near the $U B$ bands, and the spectrum of PSR B0656+14 has

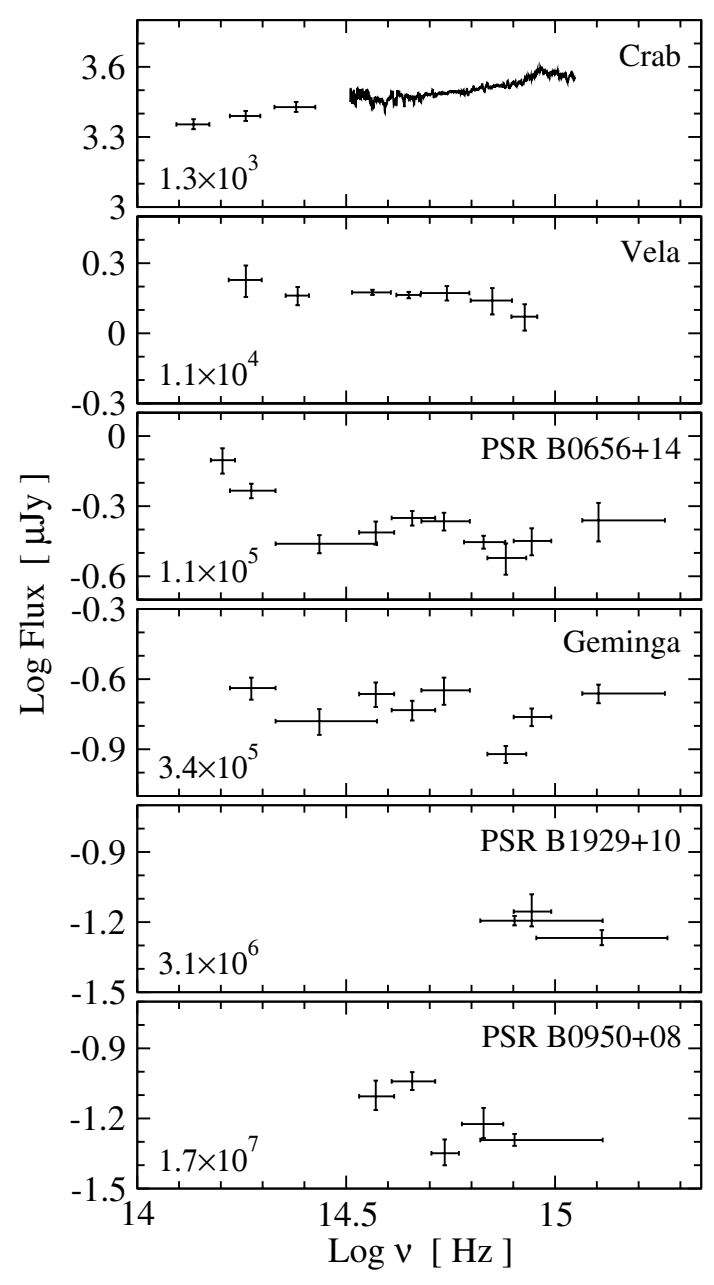

Fig. 4. Optical spectra of pulsars of different dynamical age $\tau=P / 2 \dot{P}$, which is indicated in the left-bottom corner of each panel in years, from the youngest Crab pulsar at the top to the oldest PSR B0950+08 at the bottom.

a significant increase in the near-IR range (Koptsevich et al. 2001; Komarova et al. 2003). We see signs of these features also in the Vela spectrum, although at a low significance level. In this context, the deviations from a single power law spectrum of the oldest PSR B0950+08 would be reminiscent of spectral features known to be associated with middle-aged pulsars, indicating the possible spectral evolution of the pulsar optical emission with age. However, deep optical observations of this pulsar and PSR B1929+10, which is about 6 times younger and brighter in UV but not studied yet in the optical range, would be valuable to decrease flux uncertainties and to constrain better spectral properties of old pulsars in this range. Acquiring pulsations of the PSR B0950+08 BVRI counterpart and accurate measurements of its proper motion would be important to unambiguously confirm it as the pulsar.

\subsection{Optical luminosity of PSR B0950+08 and efficiency evolution of pulsars in the optical and other spectral ranges}

It is believed that the nonthermal radiation of pulsars is powered by their rotation energy losses $\dot{E}=4 \pi^{2} I \dot{P} / P^{3}$, where 
Table 6. The ages $\tau$, distances $d$, spindown luminosities $\dot{E}$ (or $L_{\mathrm{sd}}$ ), and observed nonthermal luminosities in the radio $L_{\mathrm{R}}$, optical $L_{\mathrm{Opt}}$, X-ray $L_{\mathrm{X}}$, and gamma-ray $L_{\gamma}$ ranges of seven radio pulsars detected in the optical range.

\begin{tabular}{|c|c|c|c|c|c|c|c|}
\hline Source & $\begin{array}{c}\log \tau^{a} \\
{[\mathrm{yr}]}\end{array}$ & $\begin{array}{l}d \\
{[\mathrm{pc}]}\end{array}$ & $\begin{array}{l}\log \dot{E} \\
{\left[\operatorname{erg~s}^{-1}\right]}\end{array}$ & $\begin{array}{l}\log L_{\mathrm{R}}^{b} \\
{\left[\mathrm{mJy} \mathrm{kpc}{ }^{2}\right]} \\
408 \mathrm{MHz}\end{array}$ & $\begin{array}{l}\log L_{\mathrm{Opt}}^{c} \\
{\left[\operatorname{erg~s}^{-1}\right]} \\
B \text { band }\end{array}$ & $\begin{array}{l}\log L_{\mathrm{X}}^{d} \\
{\left[\operatorname{erg~s}^{-1}\right]} \\
2-10 \mathrm{KeV}\end{array}$ & $\begin{array}{l}\log L_{\gamma}^{e} \\
{\left[\operatorname{erg~s}^{-1}\right]} \\
\geq 100 \mathrm{MeV}\end{array}$ \\
\hline $\mathrm{Crab}$ & 3.1 & $2.0(1) \times 10^{3}$ & 38.65 & $3.41(4)$ & $32.23(5)$ & $36.67\left({ }_{-26}^{+20}\right)$ & $35.7\left({ }_{-3}^{+1}\right)$ \\
\hline B0540-69 & 3.2 & $5.00\left(\begin{array}{c}+50 \\
-6\end{array}\right) \times 10^{4}$ & 38.17 & $3.30(9)^{g}$ & $33.47(15)$ & $36.99\left({ }_{-23}^{+19}\right)$ & $\leq 35.97$ \\
\hline Vela & 4.1 & $293\left(\left(_{-17}^{+19}\right)^{f}\right.$ & 36.84 & $2.64(20)$ & $28.3(3)$ & $31.2\left(\left(_{-38}^{+36}\right)^{i}\right.$ & $33.9\left({ }_{-3}^{+1}\right)$ \\
\hline B0656+14 & 5.0 & $288\left({ }_{-27}^{+33}\right)^{f}$ & 34.58 & $-0.27(9)$ & $27.53(8)$ & $30.30\left(\left(_{-28}^{+36}\right)\right.$ & $32.37\left(\left(_{-30}^{+10}\right)\right.$ \\
\hline Geminga & 5.5 & $153\left({ }_{-34}^{+59}\right)^{f}$ & 34.51 & $0.375\left(_{-23}^{+27}\right)^{h}$ & $26.95\left(\left(_{-10}^{+16}\right)\right.$ & $29.35\left(\left(_{-36}^{+38}\right)\right.$ & $32.95\left(\left(_{-30}^{+10}\right)\right.$ \\
\hline B $1929+10^{5}$ & 6.5 & $331(10)^{f}$ & 33.59 & $1.52(5)$ & $27.26\left({ }_{-33}^{+20}\right)$ & $29.86\left(\left(_{-15}^{+13}\right)\right.$ & $\leq 32.57$ \\
\hline B0950+08 & 7.2 & $262(5)^{f}$ & 32.75 & $1.44(16)$ & $26.88(8)$ & $29.28\left(\left(_{-18}^{+13}\right)\right.$ & $\leq 32.51$ \\
\hline \multicolumn{4}{|c|}{${ }^{a}$ Dynamical age $\tau=P / 2 \dot{P}$. } & \multicolumn{4}{|c|}{${ }^{f}$ Parallax based distances: from Dodson et al. (2003) and } \\
\hline \multirow{3}{*}{\multicolumn{4}{|c|}{$\begin{array}{l}{ }^{b} \text { The pulsar fluxes are from European Pulsar Network } \\
\text { archive http://www.mpifr-bonn.mpg.de/div/pulsar/data, } \\
\text { unless specified otherwise. }\end{array}$}} & Caraveo & 1. (2001) for & ; from Brisl & t al. (2003) for \\
\hline & & & & B0656+ & rom Caravec & l. $(1996) \mathrm{f}$ & minga; from \\
\hline & & & & Brisken & (2002) for & $50+08$ and & $9+10$ \\
\hline \multicolumn{4}{|c|}{${ }^{c}$ The data are from Zharikov et al. (2002), updated } & \multicolumn{4}{|c|}{${ }^{g}$ From Crawford et al. (2001). } \\
\hline \multicolumn{4}{|c|}{ with new distance estimates. } & \multicolumn{4}{|c|}{${ }^{h}$ Only a flux upper limit is known at $408 \mathrm{MHz}$; a mean } \\
\hline \multicolumn{4}{|c|}{$\begin{array}{l}{ }^{d} \text { Nonthermal fluxes are from Possenti et al. (2002), } \\
\text { unless specified otherwise. }\end{array}$} & \multicolumn{4}{|c|}{$\begin{array}{l}\text { flux at } 102 \mathrm{MHz} \text { (Kassim \& Lazio } 1999 \text { and refs therein) is } \\
\text { used instead of that. }\end{array}$} \\
\hline \multicolumn{4}{|c|}{$\begin{array}{l}{ }^{e} \text { Based on the EGRET data from Thompson (2000) and } \\
\text { Thompson et al. (1994), updated with new distances. }\end{array}$} & \multicolumn{4}{|c|}{$\begin{array}{l}{ }^{i} \text { Based on recent Chandra data (Pavlov et al. 2001), } \\
\text { updated with new Vela distance. }\end{array}$} \\
\hline
\end{tabular}

$I=10^{45} \mathrm{gm} \mathrm{cm}^{-2}$ is the inertia momentum of a NS of 1.4 sollar mass and $10 \mathrm{~km}$ radius. $\dot{E}$ is frequently called also the spindown luminosity and denoted as $L_{\mathrm{sd}}\left(L_{\mathrm{sd}} \equiv \dot{E}\right)$. The ratio $\eta=L / \dot{E}$, where $L$ is the photon luminosity, is generally used to characterize the efficiency of the $\dot{E}$ conversion into the pulsar emission at a given spectral domain (e.g., Goldoni et al. 1995). To estimate the optical efficiency of PSR B0950+08 we calculated the integrated unabsorbed flux of the pulsar in 4000-9000 $\AA$ range $F_{\text {Opt }} \approx 4.2 \times$ $10^{-14} \mathrm{ergs} \mathrm{cm}^{-2} \mathrm{~s}^{-1}$. This suggests the optical luminosity $L_{\mathrm{Opt}} \simeq 3.5(7) \times 10^{27}(\mathrm{~d} / 262 \mathrm{pc})^{2} \mathrm{erg} \mathrm{s}^{-1}$ and the optical efficiency $\eta_{\mathrm{Opt}}=L_{\mathrm{Opt}} / \dot{E} \simeq 6.5(5) \times 10^{-6}$. Scaled to the $B$ band $F W H M$, this value is consistent with previous estimates of the efficiency based on the Subaru observations in only the $B$ band (Zharikov et al. 2002). It is much higher than the efficiency of middle-aged pulsars and comparable to that of the young and energetic Crab pulsar. In this context, PSR B0950+08 is not unique, another old pulsar PSR B1929+10 is characterized with almost the same efficiency. This implies that pulsars may become efficient optical emitters again before the end of their lives, and that other old pulsars may be promising targets to search for their emission in the optical range.

The optical fluxes of PSR B0950+08 and some other pulsars detected in the optical and X-rays (e.g., Vela, PSR B0656+14, PSR B1055+10, PSR B1929+10) appear to coincide with the low energy extension of their nonthermal spectra observed in X-rays (Shibanov et al. 2003; Koptsevich et al. 2001; Pavlov et al. 2001; Mignani \& Caraveo 2001). This suggests the same nature of the nonthermal spectral components seen in the optical and X-rays. If this is true, old pulsars have to be also more efficient emitters in X-rays, as they are in the optical.

To check this we analyzed the optical and X-ray luminosities of seven pulsars of different ages detected in the optical range (Table 6). The advantage of this sample is the small uncertainty of the distances to the pulsars, $\$ 10 \%$, determined mainly by the radio or optical parallax. This minimizes errors of the inferred luminosities. Distances for other sources are much less accurate (e.g., as in case of PSR B1055-52), or their optical identifications are still questionable (e.g., as for PSR 1509-58 and PSR B1706-44), and we excluded them from our consideration. For the optical luminosities we used the $B$ band fluxes since practically all the pulsars from our set were detected in this band and the fluxes in other bands are not strongly different (cf. Fig. 4) to change significantly the relative behavior of integral optical characteristics. For X-rays we used the $2-10 \mathrm{keV}$ range where the nonthermal power law tails dominate over the thermal spectral components detected from middle-aged pulsars in a softer energy range. Most of the 2-10 keV fluxes are taken from Possenti et al. (2002). For comparison we included also the radio and gamma-ray luminosities in the $408 \mathrm{MHz}$ radio band, standard for the pulsar observations, and in the EGRET energy range ( $\gtrsim 100 \mathrm{MeV})$, respectively. The radio data are presented in units usually used in radio pulsar astronomy, $L_{\mathrm{R}}=S_{408} d_{1}^{2}\left[\mu \mathrm{Jy} \mathrm{kpc}^{2}\right]$, where $S_{408}$ is the observed flux density from a pulsar at $408 \mathrm{MHz}$, and $d_{1}$

\footnotetext{
5 Recent VLBA $5 \mathrm{GHz}$ parallax based distance measurements of B1929+10 (Chatterjee et al. 2003) lead to $d=361_{-8}^{+10} \mathrm{pc}$. The difference between this and the quoted value is only at the 2-sigma level which is insignificant for our analysis.
} 


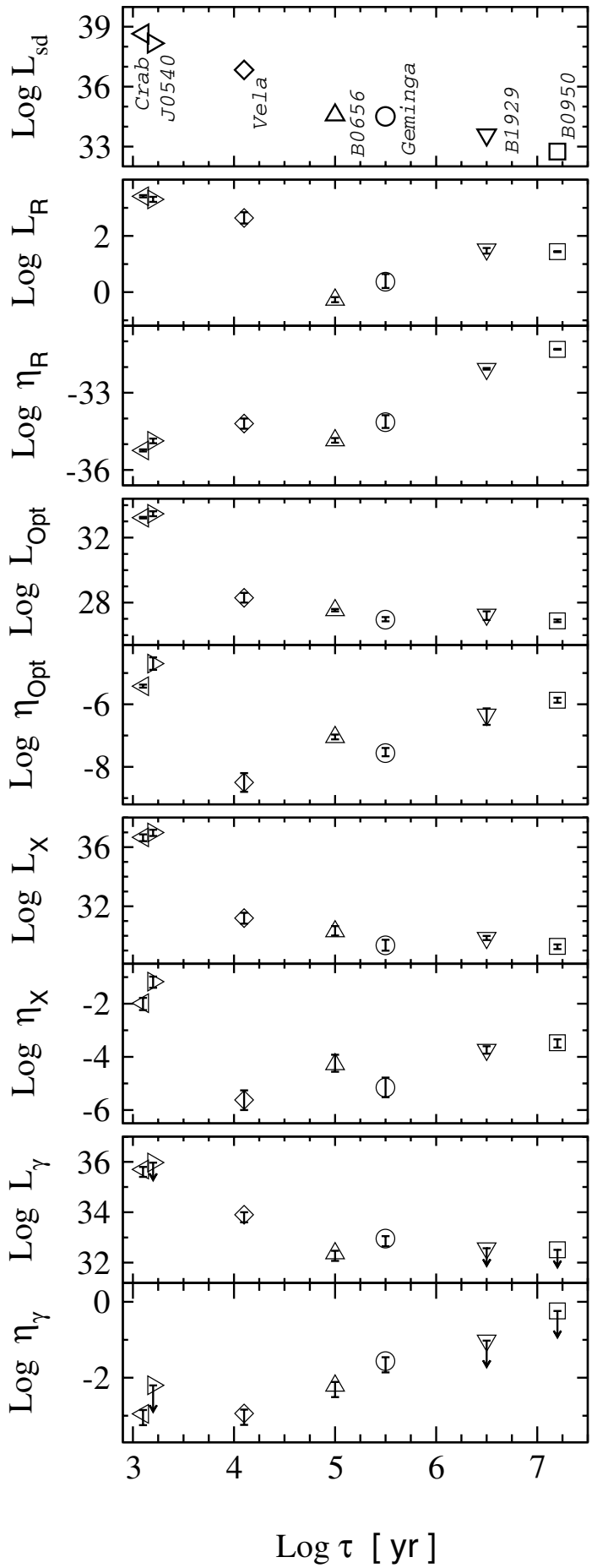

Fig. 5. From top to bottom: the evolution of the pulsar spindown, radio, optical, X-ray, and gamma-ray luminosities $\left(L_{\mathrm{sd}}, L_{\mathrm{R}}, L_{\mathrm{Opt}}, L_{\mathrm{X}}\right.$, and $L_{\gamma}$ ) and respective efficiencies, $\eta_{\mathrm{R}}, \eta_{\mathrm{Opt}}, \eta_{\mathrm{X}}, \eta_{\gamma}$, with the dynamical age $\tau$ based on the data presented in Table 6. Each pulsar is denoted by a certain symbol, as shown at the top panel; error bars correspond to $\pm 1 \sigma$ uncertainties of the values.

is its distance in kpc. At the typical radio band $F W H M$ of about $100 \mathrm{kHz}$ the conversion coefficient to standard units is $\approx 9.61 \times 10^{21}\left[\mathrm{erg} \mathrm{s}^{-1}\right] /\left[\mu \mathrm{Jy} \mathrm{kpc}^{2}\right]$.

In Fig. 5 we plotted the pulsar luminosities in the mentioned above ranges and respective efficiencies versus the dynamical age $\tau$. As seen, the optical and X-ray luminosities

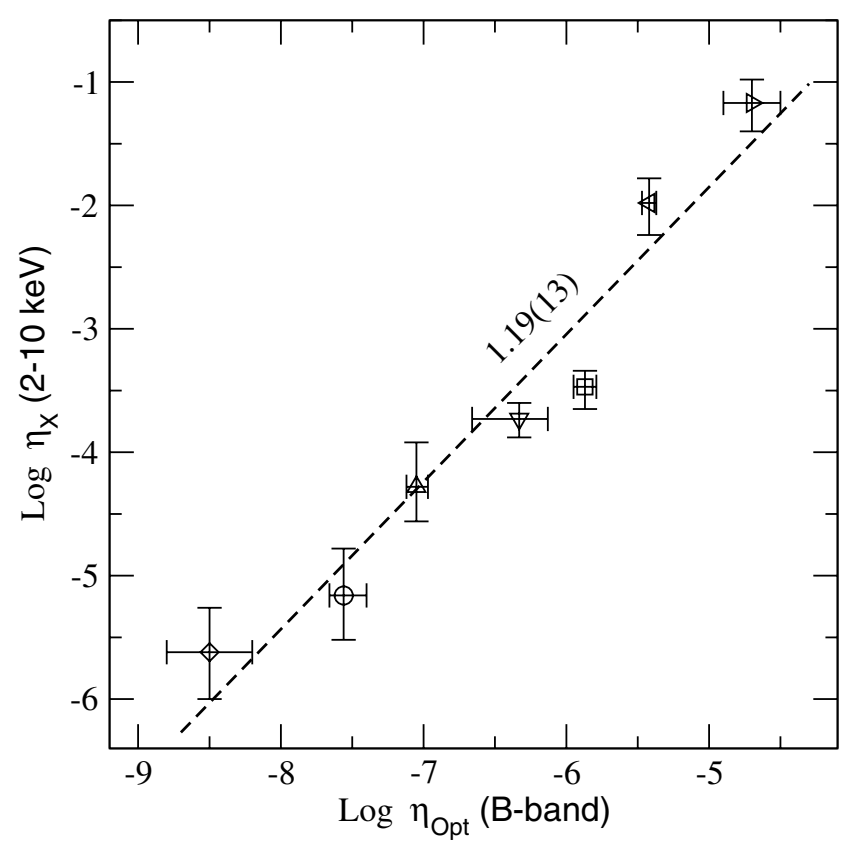

Fig. 6. Relationship between the optical ( $\left.\eta_{\mathrm{Opt}}\right)$ and X-ray $\left(\eta_{\mathrm{X}}\right)$ efficiencies in the $B$ band and $2-10 \mathrm{keV}$ ranges, respectively. Different pulsars are denoted by the same symbols, as in Fig. 5. The dashed-line shows the best fit linear model with the slope value indicated near the line.

decrease monotonously with age until $\tau \sim 10^{4}-10^{5} \mathrm{yr}$, and after that they become almost constant, while the spindown luminosity continues to decrease. The dependences of $L_{\mathrm{Opt}}(\tau)$ and $L_{\mathrm{X}}(\tau)$, as well as $\eta_{\mathrm{Opt}}(\tau)$ and $\eta_{\mathrm{X}}(\tau)$, are remarkably similar, and old pulsars are indeed also efficient X-ray emitters, as in the optical range, with the efficiencies close to those of young and energetic Crab-like pulsars.

The behavior of the radio luminosity is less monotonic but it starts to increase gradually with $\tau$ approximately from the same age, as $L_{\mathrm{Opt}}(\tau)$ and $L_{\mathrm{X}}(\tau)$ become constants. Older PSR B1929+10 and PSR B0950+08 are about two orders of magnitude more luminous in the radio than the middle-aged PSR B0656+14. This increase is even more pronounced in the efficiencies, demonstrating that old pulsars from our sample are also much more efficient radio emitters than younger ones, at least in the considered $408 \mathrm{MHz}$ band. The same appears to be true in the $\gamma$-ray range, although only upper flux limits are known in the EGRET range for two oldest pulsars from the set.

A linear regression fit of the optical and X-ray efficiencies, taking into account uncertainties in both values, yields

$\log \eta_{\mathrm{X}}=4.12(0.87)+1.19(0.13) \log \eta_{\text {Opt }}$,

with a correlation coefficient of 0.97 . The fit is shown by the dashed line in Fig. 6. This is additional evidence for an origin of the nonthermal spectral components in both ranges, independent of the pulsar age and luminosity levels.

The relationship between the radio and gamma-ray efficiencies apparently seen in Fig. 5 is much less plausible. The linear model fit is too uncertain in this case,

$\log \eta_{\gamma}=17.05(27.53)+0.56(0.79) \log \eta_{\mathrm{R}}$,

with a correlation coefficient only of 0.44 , albeit consistent with the gamma-ray flux upper limits of three pulsars in the set (see 


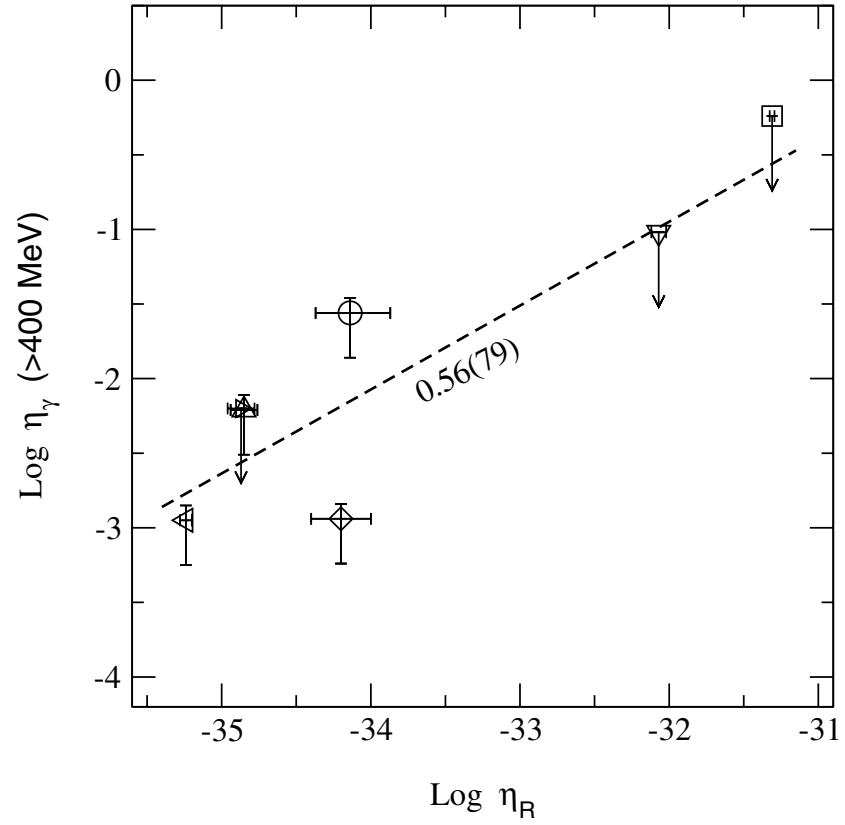

Fig. 7. The same as in Fig. 6, but for the radio $\left(\eta_{\mathrm{R}}\right.$ at $\left.408 \mathrm{MHz}\right)$ and gamma-ray $\left(\eta_{\gamma}\right)$ efficiencies.

Fig. 7). We have not found any significant global correlations between the optical and radio, optical and gamma, as well as between X-rays and radio, and X-rays and $\gamma$-rays in the considered sample of pulsars.

As it has been noted by several authors (e.g., see Brisken et al. 2003 and refs therein), the dynamical age of a $10 \mathrm{Myr}$ pulsar can differ by $\sim 40 \%$ from its true age. However, the ages of younger pulsars in our set are much more accurate since they are consistent with the ages of associated supernova remnants, and even larger, e.g., a factor of $\sim 2$, uncertainties in the ages of the old PSR B1929-10 and PSR B0950+08 can barely change qualitatively $\tau$-dependencies shown in Fig. 5. Non-monotonic behavior of the evolution tracks $\eta_{\mathrm{Opt}}(\tau)$ and $\eta_{\mathrm{X}}(\tau)$ with the pronounced minimum at the beginning of a middle-aged epoch is a new fact, whose physical origin has yet to be understood. Rising branches of the tracks appear to coincide with a steep increase of $\eta_{\mathrm{R}}(\tau)$ and less definite increase of $\eta_{\gamma}(\tau)$.

Figure 8, where we plotted efficiencies versus the spindown luminosity $L_{\mathrm{sd}}$, provide a physical insight into the problem. The linear regression fit of the X-ray efficiency of the whole sample of pulsars,

$\log \eta_{\mathrm{X}}=-15.9(9.7)+0.34(0.27) \log L_{\mathrm{sd}}$,

is shown by the dashed line in the respective panel of Fig. 8. Even within large uncertainties caused mainly by limited data set of our sample, this fit is consistent with that of Possenti et al. (2002),

$\log \eta_{\mathrm{X}}=-15.34+0.34 \log L_{\mathrm{sd}}$,

based on much large number of pulsars detected in X-rays. Formally our fit is statistically unacceptable. Nevertheless, we see the two youngest individuals, Crab pulsar and PSR B0540-69, located definitely away from the rest set in the plots of $\eta_{\mathrm{Opt}}\left(L_{\mathrm{sd}}\right)$ and $\eta_{\mathrm{X}}\left(L_{\mathrm{sd}}\right)$. Exclusion of these members

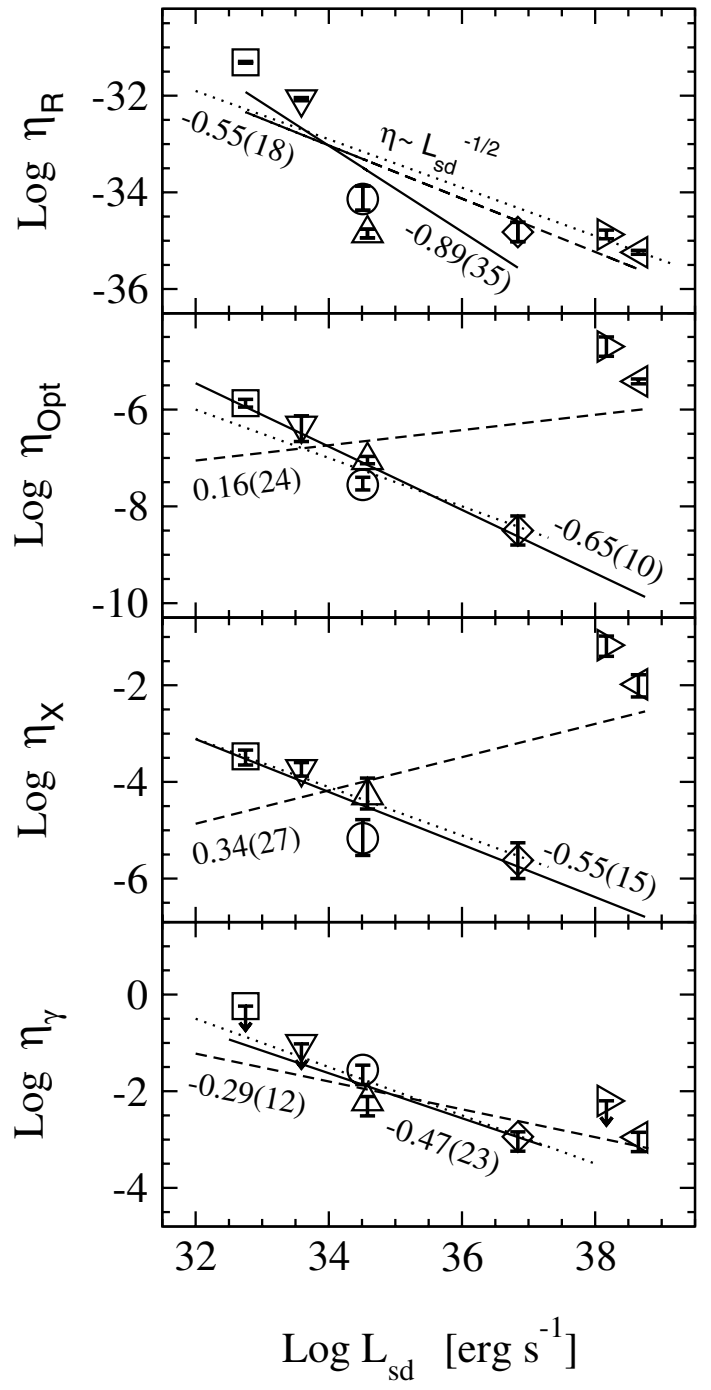

Fig. 8. From top to bottom: relationships between the efficiencies in the radio, optical, $\mathrm{X}$-ray, and $\gamma$-ray spectral domains and the spindown luminosity $L_{\text {sd }}$. Different pulsars are denoted by the same symbols, as in Figs. 5-7. Dashed and solid lines show the best linear regression fits of the whole set of pulsars, and without two younger ones (Crab and PSR B0540-69, with the highest $L_{\text {sd }}$ ), respectively. The slope values are indicated near the lines. Dotted lines in all the panels indicate a slope in case of the linear proportionality of the luminosities to the Goldreich-Julian current.

yields much better fits with significantly negative slopes in both spectral domains,

$\log \eta_{\mathrm{X}}=14.3(5.0)-0.55(0.15) \log L_{\mathrm{sd}}$,

$\log \eta_{\mathrm{Opt}}=15.5(3.4)-0.65(0.10) \log L_{\mathrm{sd}}$.

These fits are shown by solid lines and they underline a branch of middle-aged-old pulsars whose optical and X-ray efficiencies increase with the spindown luminosity decrease. Within uncertainties the solid line slopes in both spectral domains are consistent with each other. For convenience of the visual comparison the log-scales of vertical axes in Fig. 8 are approximately the same in all the panels.

The Crab and PSR B0540-69 do not look significantly exceptional in the $\eta_{\mathrm{R}}\left(L_{\mathrm{sd}}\right)$ and $\eta_{\gamma}\left(L_{\mathrm{sd}}\right)$ panels. The efficiency 
distributions in these spectral domains show negative slopes everywhere in the considered range of $L_{\mathrm{sd}}$. The slopes are apparently less steep at higher $L_{\mathrm{sd}}$. Although the efficiency upper limits for three pulsars are not included in the fits in the gammaray range, they are consistent with the derived dependences.

Based on the results presented in Fig. 8, it is natural to assume that in all spectral domains the emission of the pulsar from the subsamples with negative slopes is regulated by the same physical process converting $L_{\mathrm{sd}}$ into the observed radiation. An additional factor is switched on to amplify significantly the efficiency of young pulsars in the optical-X-ray range, and, possible, to maintain it at a certain level in the radio and $\gamma$-rays.

Excluding the two youngest pulsars from the set, the optical-X-ray efficiency of the pulsars in our sample is approximately $\propto L_{\mathrm{sd}}^{-1 / 2}$ (dotted lines in Fig. 8). This means that the luminosity is proportional to $L_{\mathrm{sd}}^{+1 / 2}$, or to the pulsar open field line voltage $V$, which, in turn, is proportional to the polar cap (Goldreich-Julian) current. The latter has long been recognized as a significant physical parameter for high energy emission of pulsars (e.g., Arons 1996). We see that the linear proportionality of the luminosity to the voltage works almost perfectly in the optical and X-ray spectral domains for not too young pulsars from our set. The spectral indices $\Gamma$ of nonthermal spectral components (with the spectral fluxes $F_{E} \propto E^{-\Gamma}, E$ is the photon energy) detected in $2-10 \mathrm{keV}$ range for a population of young Crab-like pulsars show the same trend, but with the reverse sign: $\Gamma \approx \Gamma_{\max }-c L_{\mathrm{sd}}^{-1 / 2}$, where $\Gamma_{\max } \approx 2$ is an observational upper limit of the spectral slopes over the sample, and $c$ is a positive constant (Gotthelf 2003). Although possible connection of a lower efficiency with a higher index or steeper and softer spectrum does not look physically unreasonable, it is not clear yet whether this is true for our subsample, since spectral indices for older pulsars considered here are still rather uncertain.

The linear proportionality of the luminosity to the open field line voltage, which is responsible for the acceleration of relativistic particles emitting nonthermal radiation, is likely seen for younger pulsars in the radio (dashed line in the upper panel of Fig. 8), and for the older pulsar subset in gamma-rays (solid line in the bottom panel). On the other hand, the luminosity of the older subset is roughly $\propto V^{0.2}$ in the radio (corresponding to the solid line in the upper panel), and the luminosity of a younger subset is $\propto V^{1.4}$ in gamma-rays (corresponding to the dashed line in the bottom panel). This indicates that the radio luminosity of older pulsars is less sensitive to the voltage drop with age, and these pulsars use their smaller $V$ value with higher efficiency in this range than younger ones. In $\gamma$-rays younger pulsars possibly demonstrate a stronger dependence on $V$ at a significantly higher voltage value in comparison with older pulsars, although derived uncertainties in this range are too large to make definite conclusions. At the same time, the latter statement appears to have much higher significance in the optical-X-ray domain, where two young pulsars are significantly apart from the older population.

The evolutionary trends and relationships presented herein are based only on a limited sample of the pulsars believed to be reliably detected in the optical range. Nevertheless, the well determined distances to the objects together with the bulk of available data in other spectral domains obtained by independent methods partially compensate poor object statistics. With some modifications many features are reproduced in different spectral ranges. This is encouraging and lends credibility to the results to take them into account in further multiwavelength studies of pulsars, including the optical range, to choose promising targets to search for new pulsar counterparts to complete the sample, to test and tune suggested evolution features and relationships, and to construct finally a unified model of multiwavelength emission of rotation powered NSs. New generation large ground-based telescopes like the VLT and Subaru can contribute significantly to these studies by the acquisition high quality data on pulsars previously identified in the optical range and by the search for new identifications.

\section{Conclusion}

The most important results of our study can be summarized as follows.

1. The candidate to the optical counterpart of PSR B0950+08 was studied, for the first time, in the $V R I$ bands. We also confirmed its detection in the $B$ band. Unusual colors and the positional coincidence of the object with the radio position strongly suggest that we detected the optical counterpart of the pulsar.

2. Our VLT observations confirm a $20 \%$ elongation of the counterpart spatial profile perpendicular to the pulsar proper motion in the $B$ band, detected earlier with the Subaru telescope (Zharikov et al. 2002). This may indicate the presence of a compact pulsar nebula. We found also two faint red background objects, likely distant galaxies, in $\sim 1$ '. 2 vicinity of the pulsar.

3. The broadband spectrum of the optical counterpart can be described by a single power law with a negative slope suggesting nonthermal nature of the pulsar optical emission. Possible spectral excesses and depressions cannot be excluded. This makes the pulsar by its optical spectral properties closer to the middle-aged pulsars.

4. We found a significant correlation, with a correlation coefficient of 0.97 , between the nonthermal optical and $2-10 \mathrm{keV}$ X-ray luminosities of isolated pulsars detected in the optical range. This provides additional evidence for the origin of the emission in both spectral domains, as has been noticed from a direct comparison of the optical and X-ray spectra of some middle-aged pulsars (e.g., Koptsevich et al. 2001).

5. The evolution of the optical and X-ray efficiencies of these pulsars shows non-monotonic behavior with a minimum at the middle-aged epoch. Old pulsars have almost the same efficiency as the young and energetic Crab-like objects. In accordance with that, the young Crab-like pulsars appear to demonstrate much steeper proportionality of the luminosities to the pulsar open magnetic field line voltage than the subpopulation of older ones, although the statistics of the objects is still very limited to make definite conclusions.

Acknowledgements. This work has been partially supported by CONACYT project 25454-E and 36585-E, and RFBR 
grants 02-02-17668, 03-02-17423, and 03-07-90200, and DIUC 202.011.030-1.0. We used the USNOFS Image and Catalogue Archive operated by the United States Naval Observatory, Flagstaff Station (http://www.nofs.navy.mil/data/fchpix/). We are grateful to the anonymous referee for many useful comments which allowed us to improve considerably the text.

\section{References}

Arons, J. A. 1996, A\&AS, 120, 49

Bessel, M. S. 1990, PASP, 102, 1181

Brisken, W. F., Benson, J. M., Goss, W. M., et al. 2002, ApJ, 571, 906

Brisken, W. F., Fruchter, A. S., Goss, W. M., et al. 2003, ApJ, accepted [astro-ph/0306232]

Caraveo, P. A., Bignami, G. F., Mignani, R., et al. 1996, ApJ, 461, L91

Caraveo, P. A., De Luca, A., Mignani, R. P., et al. 2001, ApJ, 561, 930

Chatterjee, S., Cordes, J. M., Vlemmings, W. H. T., et al. 2003 [astro-ph/0312044]

Cordova, F. A., Hjellming, R. M., Mason, K. O., \& Middleditch, J. 1989, ApJ, 345, 451

Crawford, F., Kaspi, V. M., Manchester, R. N., et al. 2001, ApJ, 553, 367

Dodson, R., Legge, D., Reynolds, J. E., et al. 2003, ApJ, accepted [astro-ph/0302374]

Fomalont, E. B., Goss, W. M., Lyne, A. G., et al. 1992, MNRAS, 258, 497

Fukugita, M., Shimasaku, K., \& Ichikawa, T. 1995, PASP, 107, 945

Goldoni, P., Musso, C., Caraveo, P. A., et al. 1995, A\&A, 298, 535

Gotthelf, E. V. 2003, ApJ, 591, 361

Hill, R. J., Dolan, J. F., Bless, R. C., et al. 1997, ApJ, 486, L99

Kassim, N., \& Lazio, T. J. 1999, ApJ, 527, L101
Komarova, V. N., Shibanov, Yu. A., Zharikov, S. V., et al. 2003; in press Pulsars, AXPs and SGRs observed with BEPPOSAX and other observatories Marsala (Sicily) 23-25 Sep. 2002

Koptsevich, A. B., Pavlov, G. G., Zharikov, S. V., et al. 2001, A\&A, 370,1004

Kurt, V. G., Sokolov, V. V., Zharikov, S. V., et al. 1998, A\&A, 333, 547

Kurt, V. G., Komarova, V. N., Fatkhullin, T. A., et al. 2000, BSAO, 49, 5

Landolt, A. 1992, AJ, 104, 340

Manning, R. A., \& Willmore, A. P. 1994, MNRAS, 266, 635

Martin, C., Halpern, J. P., \& Schiminovich, D. 1998, ApJ, 494, L211

Mignani, R. P., \& Caraveo, P. A. 2001, A\&A, 376, 213

Pavlov, G. G., Stringfellow, G. S., \& Cordova, F. A. 1996, ApJ, 467, 370

Pavlov, G. G., Zavlin, V. E., Sunwal, D., et al. 2001, ApJ, 552, L129

Possenti, A., Cerutti, R., Colpi, M., et al. 2002, A\&A, 387, 993

Seward, F., \& Wang, Z. 1988, ApJ, 332, 199

Sokolov, V. V., Kurt, V. G., Zharikov, S. V., et al. 1998, The 19th Texas Symposium on Relativistic Astrophysics and Cosmology, held in Paris, France, Dec. 14-18, 1998, ed. J. Paul, T. Montmerle, \& E. Aubourg (CEA Saclay)

Shibanov, Yu. A., Koptsevich, A. B., Solleram, J., et al. 2003, A\&A, 406,645

Sollerman, J., Lundqvist, P. Lindler, D., et al. 2000, ApJ, 537, 861

Sollerman, J. 2003, A\&A, 406, 639

Taylor, J. H., Manchester, R. N., \& Lyne, A. G. 1993, ApJS, 88, 529

Thompson, D. J., Arzoumanian, Z., Bertsch, D. L., et al. 1994, ApJ, 436, 229

Thompson, D. J. 2000, AdSpR, 25, 659

Zharikov, S. V., Shibanov, Yu. A., Koptsevich, A. B., et al. 2002, A\&A, 394, 633 